\title{
Novedades metodológicas aplicadas a la antropología alimentaria: modelos basados en agentes y redes sociales
}

\author{
Methodological novelties applied to the anthropology of \\ food: agent-based models and social networks analysis
}

${ }^{1}$ Doctor en Ciencias Antropológicas. Investigador Instituto de Salud Colectiva, Universidad Nacional de Lanús, Argentina. $\triangle$ (iD)
RESUMEN En este artículo presentamos dos modalidades metodológicas que aún no han sido muy utilizadas en la antropología alimentaria. Por un lado, nos referimos al análisis de redes sociales y, por otro, a los modelos basados en agentes. Para ilustrar los métodos, tomaremos dos casos de materiales clásicos de la antropología alimentaria. Para el primero usaremos los platos de comida de un relevamiento hecho en la Quebrada de Humahuaca (provincia de Jujuy, Argentina) y, para el segundo, utilizaremos algunos elementos del concepto aplicado por Aguirre de "estrategias domésticas de consumo". La idea subyacente es que, dado que la alimentación se reconoce como un "hecho social total" y, por lo tanto, como un fenómeno complejo, el abordaje metodológico debe seguir necesariamente esa misma característica. Mientras más métodos utilicemos (con el grado de rigor adecuado) mejor estaremos preparados para comprender la dinámica alimentaria en el medio social.

PALABRAS CLAVES Antropología; Alimentación; Simulación por Computadora; Dinámicas no Lineales.

\begin{abstract}
The aim of this article is to introduce two methodological strategies that have not often been utilized in the anthropology of food: agent-based models and social networks analysis. In order to illustrate these methods in action, two cases based in materials typical of the anthropology of food are presented. For the first strategy, fieldwork carried out in Quebrada de Humahuaca (province of Jujuy, Argentina) regarding meal recall was used, and for the second, elements of the concept of "domestic consumption strategies" applied by Aguirre were employed. The underlying idea is that, given that eating is recognized as a "total social fact" and, therefore, as a complex phenomenon, the methodological approach must also be characterized by complexity. The greater the number of methods utilized (with the appropriate rigor), the better able we will be to understand the dynamics of feeding in the social environment.
\end{abstract}

KEY WORDS Anthropology; Feeding; Computer Simulation; Nonlinear Dynamics. 


\section{INTRODUCCIÓN}

Como bien señalara Marcel Mauss ${ }^{(1)}$ y se convirtiera en un axioma teórico, la alimentación es un "hecho social complejo". Esto implica que atraviesa todos los aspectos de lo social (económico, religioso, jurídico, político, etc.), pero también los aspectos de base, es decir, fisiológicos y psicológicos, y todo en forma simultánea. La alimentación, para los seres humanos, es un problema que abarca desde la molécula hasta el símbolo. Pero en su accionar cotidiano, la alimentación es un fenómeno que se repite más de una vez por día, se percibe como algo completo y total, incluso oscureciendo sus orígenes y causas; por tanto, la división planteada es puramente analítica.

Si la alimentación es un fenómeno complejo, entonces también lo tienen que ser las herramientas metodológicas con las que se lo intenta describir (o explicar, sin ánimo de entrar en esa discusión epistemológica). En ese sentido, siempre sostuvimos que en antropología alimentaria es necesario utilizar tanto los métodos cuantitativos como los cualitativos, ya que cada uno ilumina diferentes aspectos del problema y, si se los combina adecuadamente, se logra una comprensión más acabada del fenómeno.

En este artículo queremos introducir dos herramientas metodológicas que pueden brindar aportes significativos al estudio antropológico de la alimentación. Por un lado, nos referimos al análisis de redes sociales, que es una metodología relacional, a diferencia de los análisis clásicos que son atributivos en los que se definen las unidades de análisis y se predican cualidades de ellas ${ }^{(2)}$. Esta metodología, se basa en los vínculos entre los nodos, por lo que el enfoque es netamente diferente. Por el otro, nos referimos a los modelos basados en agentes, que son modelos de simulación en computadora $^{(3)}$ que permiten tener un conocimiento más acabado de la temática investigada y probar escenarios diferentes aplicando la cláusula caeteris paribus (toda una novedad en ciencias sociales, al menos fuera del rango de las clásicas metodologías cuantitativas).
Como las herramientas metodológicas dependen directamente del marco teórico, tendremos que reflexionar sobre los diversos puntos de vista del uso del análisis de redes sociales o de los modelos basados en agentes. Dicho de otro modo, cualquiera de las metodologías que usemos exige una mirada particular, una forma de entender el mundo, que es la que le da el sentido. Respecto de la teoría, la clave está en la correcta identificación de sus niveles: habrá hipótesis de nivel general y otras de rango medio ${ }^{(4)}$. La metodología suele ser la que vincula las teorías de rango medio con la empiria, ya que la teoría general es la encargada de dar los fundamentos principales, la que explicita los supuestos. En el caso del análisis de redes sociales, lo importante es identificar las relaciones que rigen a los actores ${ }^{(2)}$. En el caso de los modelos basados en agentes, lo importante es identificar las reglas que los actores sociales utilizan para su acción ${ }^{(3)}$. En el primer caso, el énfasis está puesto en el carácter relacional (las relaciones sociales elegidas); en el segundo caso, el énfasis está puesto en lo normativo (desde el punto de vista del propio actor).

En este artículo presentaremos dos casos que pueden ilustrar las ventajas y desventajas de usar esta clase de metodologías. En cuanto al dominio técnico necesario para poder usarlas, lo importante no es el conocimiento tecnológico, sino la forma de pensar y abordar los problemas científicos. Podríamos decir que el conocimiento tecnológico es, en algún sentido, espurio; lo verdaderamente difícil es cambiar la forma de pensar y comprender que el foco se coloca en las relaciones, en el caso del análisis de redes sociales ${ }^{(2)}$, y en la abstracción de las reglas de conducta, en el caso de los modelos basados en agentes ${ }^{(5)}$. El objetivo es, pues, enteramente didáctico, con la intención de tentar al investigador curioso a saborear estas nuevas metodologías, a conocerlas para, en caso de querer rechazarlas, hacerlo con fundamentos concretos y no sobre la base de prejuicios o efectos de la moda.

Por último, queremos señalar que estas metodologías se inscriben dentro de lo que 
se conoce como teoría de la complejidad y teoría del caos. Estas teorías, que son la actualización de las clásicas teorías sistémicas, conforman lo que se denomina "algoritmos de la complejidad"(6), es decir, son expresiones algorítmicas de fenómenos complejos o caóticos. Las redes, con sus comportamientos de "mundo pequeño" o su configuración como "redes libres de escala"(7), permiten apreciar (en su acepción más amplia), toda una serie de fenómenos, que otrora fueran intuidos pero no formalizados. Los modelos basados en agentes permiten observar cómo se generan comportamientos emergentes, es decir, propiedades que no pueden ser deducidas de sus condiciones iniciales y que son muy sensibles ya que reaccionan a la totalidad de actores, relaciones y contexto. Estas teorías y sus correspondientes metodologías son inespecíficas, en el sentido de que permiten tratar muchas clases diferentes de problemas, independientemente de su semántica. Se ubican, en este sentido, dentro de los conceptos teóricos de rango medio, es decir que requieren, para su correcta interpretación, de un conjunto de hipótesis que le dan especificidad. En este caso, dependen de los conceptos generales de una antropología alimentaria. Profundizaremos sobre esto en los apartados correspondientes a cada una de las metodologías presentadas.

Los datos empíricos que ilustran este artículo fueron tomados de mi tesis de doctorado $^{(8)}$ y están basados en los trabajos de campo realizados entre el año 2005 y 2010 en la Quebrada de Humahuaca, región ubicada en la provincia de Jujuy, en el noroeste argentino, que se caracteriza por estar encajonada entre los cordones Oriental y Occidental de la Cordillera de los Andes. El sentido de la Quebrada es sur-norte, elevándose hacia la región septentrional, hasta encontrarse con lo que se denomina Altiplano o Puna. El poblamiento humano de la zona posee más de 10.000 años de antigüedad $^{(9)}$ y la adopción de la agricultura tiene 4.000 años $^{(10)}$.

\section{ANÁLISIS DE REDES SOCIALES}

Antes de comenzar con nuestro ejemplo de redes sociales creemos necesario establecer algunas distinciones importantes. El concepto de redes está sumamente extendido, sobre todo a partir del uso de plataformas web que se denominan redes sociales. Pero la metodología que presentamos aquí poco tiene que ver con Facebook, Twitter o LinkedIn, aunque estos sitios web pueden ser usados como objeto de estudio. Nosotros preferimos hablar de estas plataformas como ciber redes.

En general, se reconocen tres usos para el concepto de red. En primer lugar, el sentido metafórico. En la bibliografía científica se suele hablar de redes para hacer referencia a un grupo más o menos cohesionado, en general de personas, que tienen ciertos vínculos entre $s^{\prime(11)}$. Esta forma de utilización no profundiza en los aspectos formales de la red, sino pura y exclusivamente en los aspectos metafóricos: se asume una red, pero no se indaga en ella. En segundo lugar, el sentido descrito por la palabra en inglés networking. Aquí se refiere a la posibilidad de actuar sobre una red (también definida en forma difusa, sin precisar sus componentes). Es lo que suelen hacer los que trabajan en relaciones públicas o en marketing, que se manejan en redes en forma intuitiva, sacándole provecho a la situación. En tercer lugar, la noción formal, que es la que presentamos aquí, que toma en cuenta los componentes y las características del fenómeno reticular ${ }^{(2)}$. Aquí lo que realmente importa es la estructura de la red y las medidas que pueden aplicarse sobre ella. Aquí no es importante saber (desde un punto de vista teórico) si la red está constituida por personas, instituciones, bienes o lo que sea; lo fundamental es definir los nodos y los vínculos en forma correcta.

El origen del análisis de redes sociales, en la última acepción, puede remontarse al siglo XVIII, cuando el matemático Leonard Euler estableció las bases de la teoría de grafos, a partir de un juego, clásico en su época, que consistía en cruzar los siete puentes de 
Königsberg, sin repetir ningún puente y volviendo al punto de partida. Euler encontró la solución, o podríamos decir la no-solución, ya que es imposible cruzar todos los puentes sin repetir alguno, a partir de lo cual creó la "teoría de grafos". Su solución abstracta implicó un cambio en la forma de pensar los problemas, que posteriormente se tradujo en una nomenclatura basada en vértices o nodos y aristas o vínculos ${ }^{(2)}$.

El análisis de redes sociales, en vinculación con las ciencias sociales, puede remontarse a la década de 1950, cuando el antropólogo John Barnes acuñó el término "red social", con el objetivo de estudiar las sociedades africanas y el parentesco durante el período de la descolonización ${ }^{(12)}$. Si bien el concepto y la metodología asociada no fueron muy tomadas en cuenta por la disciplina en su forma mainstream, a partir de la década de 1990, en parte como consecuencia de la posibilidad de usar computadoras personales ( $y$, por lo tanto, una enorme potencia de cálculo) y de la irrupción social de la world wide web, esta manera de abordar los estudios empezó a ser cada vez más popular (aunque hay que aclarar que todavía no forma parte del bagaje metodológico principal de las ciencias sociales).

En términos estrictamente descriptivos, una red social puede ser caracterizada como una entidad con nodos y lazos. Un nodo es simplemente una unidad que es el objeto de la vinculación. Un lazo es simplemente un vínculo entre al menos dos nodos. Qué se define como nodo y qué como lazo depende únicamente de la imaginación del investigador $^{(2)}$. En general, las unidades de análisis suelen utilizarse como nodos (sean individuos o grupos-instituciones) y los atributos vinculantes como lazos (flujos de bienes, servicios o cualquier otra entidad, entre esos nodos). A partir de esta simple definición, es posible obtener medidas de centralidad y de otro tipo que nos posibilitan vislumbrar la estructura subyacente del fenómeno y observar, por ejemplo, quién ocupa el lugar central (quién tiene más vínculos), quién conecta áreas de la red (quién funciona como puente entre dos áreas del entramado reticular) o bien qué grado de cohesión posee la red en su conjunto (cuán conectada se encuentra). Cada nodo puede tener atributos, es decir, características que lo definan más allá de sus valores reticulares. A su vez, cada lazo puede tener varias naturalezas: se puede predicar su presencia/ ausencia, se puede direccionar y se puede ponderar (es decir, cada lazo puede tener un flujo mayor o menor medido en algún tipo de unidades).

\section{El análisis de redes sociales aplicado a una red de alimentos consumidos en la Quebrada de Humahuaca}

Para la tesis de doctorado(8) decidimos usar el análisis de redes sociales y procesar los recordatorios alimentarios de 24 hs, elaborados en los trabajos de campo en la Quebrada de Humahuaca. La idea fue tratar de usar esta metodología para observar si detectábamos patrones alimentarios diferenciados entre las unidades domésticas estudiadas. En general, los recordatorios alimentarios se procesan cuantitativamente (observando frecuencias, por ejemplo) y, si bien en la tesis aplicamos esa metodología tradicional, también quisimos ver su estructura a partir del análisis de redes sociales.

Partimos de lo que se conoce como "red de dos modos". Una red de dos modos es aquella en la que las entidades son de dos tipos; por ejemplo en nuestro caso, familias y alimentos. En principio, teníamos los datos de cada unidad doméstica y los alimentos consumidos por ellos en las últimas 24 hs. Por lo tanto, no teníamos una matriz cuadrada sino una rectangular (no hay coincidencia entre el número de columnas y el número de filas). En general, para el análisis de redes sociales se recomienda usar matrices cuadradas en las que no solo coincida el número de columnas y filas en su sentido cardinal, sino en su naturaleza semántica. Es decir, las etiquetas de las columnas y de las filas deben ser las mismas. El procedimiento que utilizamos fue el clásico $^{(13)}$ : de una red de dos modos extrajimos dos redes de un modo. Es decir, 
en nuestro ejemplo, dos familias quedaban vinculadas si ambas habían comido el mismo tipo de alimento y, a la inversa, dos alimentos quedaban vinculados si una familia había consumido ambos (Figura 1).

A partir de esta matriz (aquí representada como grafo) es que construimos la red con la que trabajamos. De las dos redes que extrajimos, se trabajó únicamente con la de alimentos (Figura 2).
El grafo se distribuye de forma tal que los alimentos con mayor cantidad de lazos se encuentran en el medio y aquellos con menos vínculos se encuentran en la periferia. Una de las claves para entender este tipo de redes es que no toman en cuenta, al menos en nuestro caso, las frecuencias cuantitativas de consumo, sino que se limitan a la presencia o ausencia del vínculo. Es decir, no importa cuánto consumieron de ese alimento, sino

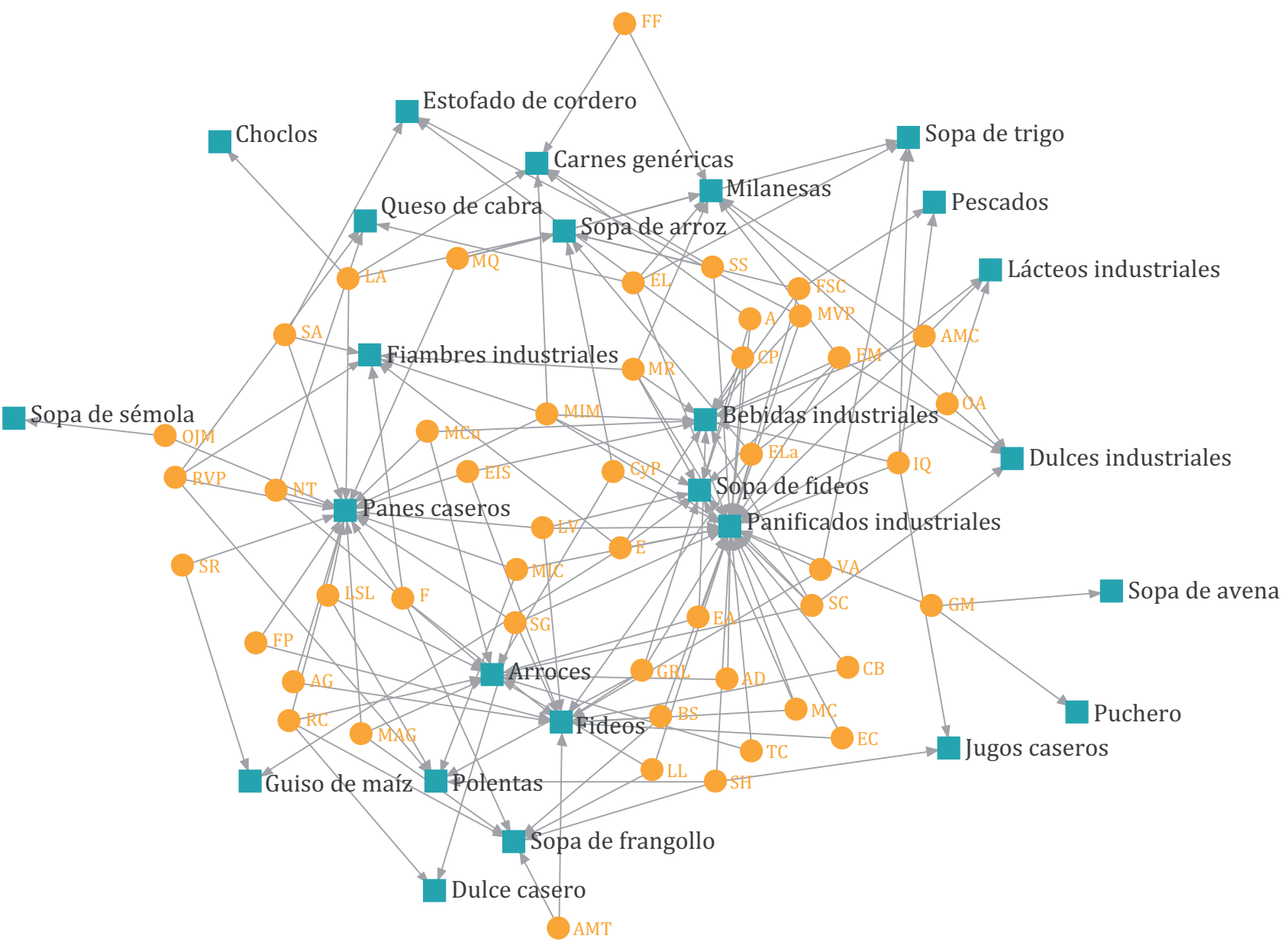

Familias Alimentos

Figura 1. Red de dos modos compuesta a partir de recordatorios de 24 hs. Quebrada de Humahuaca, Jujuy, Argentina, 2008.

Fuente: Díaz Córdova ${ }^{(8)}$. 
que lo hayan consumido. Si revisamos los datos reticulares veremos que los alimentos industriales ocupan un lugar central, pero muy cerca de ellos se encuentran los panificados caseros. Y esto refleja lo que observamos también en el trabajo de campo y que, en algún sentido, refutó una de nuestras hipótesis rectoras primigenias: los alimentos en la Quebrada de Humahuaca no se dividen en dos modalidades como habíamos supuesto - una industrial y otra local- sino que las unidades domésticas utilizan ambas, en función de las posibilidades y las conveniencias particulares.

Es decir, en este caso, el análisis de redes sociales pudo ser utilizado como una prueba extra de lo que observamos en el trabajo de campo. Las dos modalidades alimentarias (industrial y local) no pueden ser percibidas a partir de los análisis estadísticos clásicos,

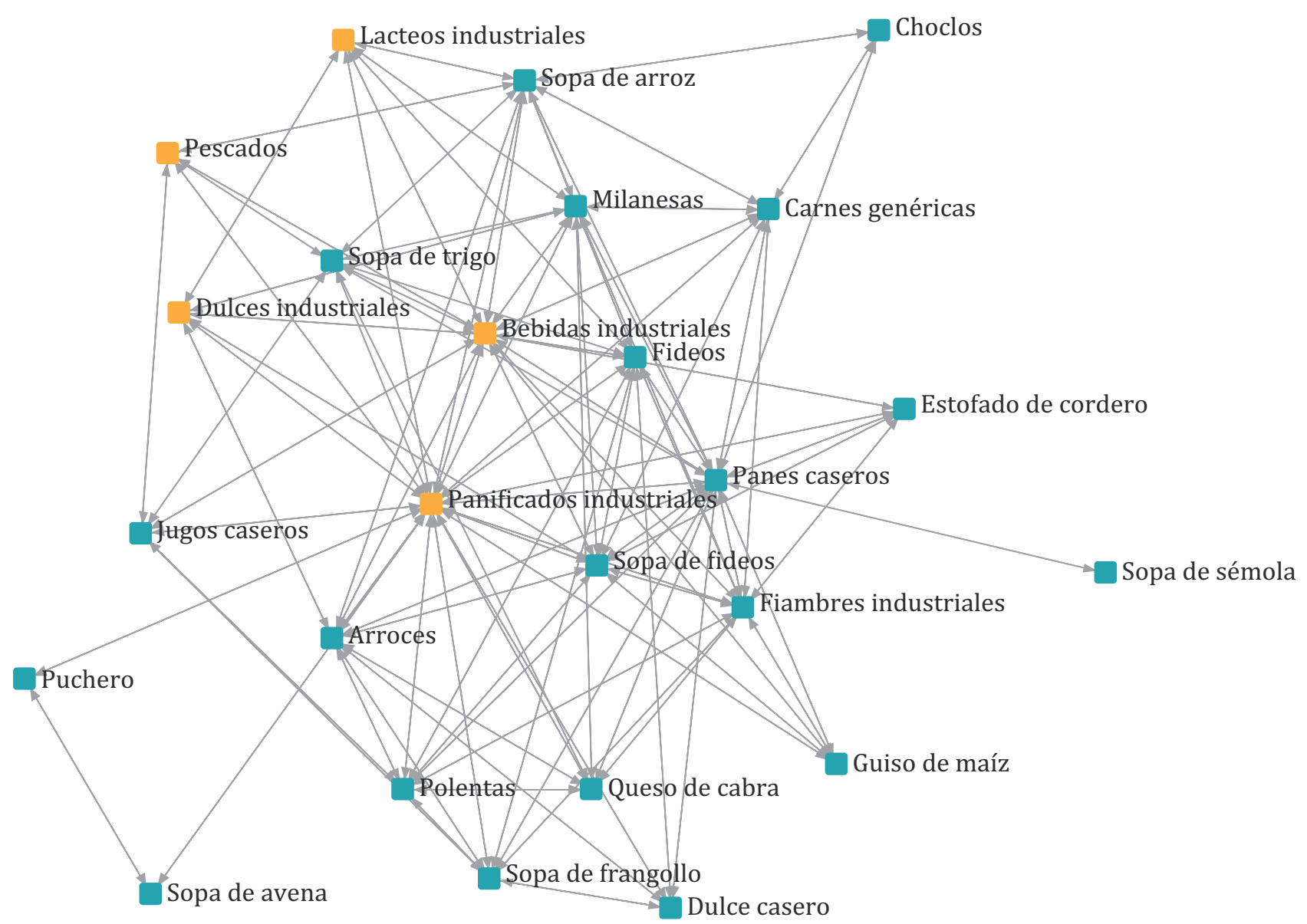

Alimentos industriales Alimentos locales

Figura 2. Red de un modo con los alimentos consumidos por las diferentes familias. Quebrada de Humahuaca, Jujuy, Argentina, 2008.

Fuente: Díaz Córdova ${ }^{(8)}$. 
fundamentalmente, porque los alimentos producidos en forma local no circulan por los mercados formales. La única manera de poder observar esa situación es haciendo el clásico trabajo de campo antropológico, del cual emerge un tipo de información nueva, vinculada con las prácticas y representaciones de los actores, y los sentidos que la gente da a sus actos alimentarios. Y si bien esto fue observado y registrado una y otra vez, como afectaba directamente a una de nuestras hipótesis fundamentales, preferimos someterla a una prueba diferente. Así fue que utilizamos el análisis de redes sociales, como una forma de observar el fenómeno desde una perspectiva independiente.

Por una cuestión de espacio no podremos presentar todas las variantes de medidas que ofrece el análisis de redes sociales. Además del grado (cantidad de vínculos), que es la que mostramos aquí, existen otras medidas que pueden ser encontradas en nuestra propia tesis de doctorado ${ }^{(8)}$, concretamente del área de la antropología alimentaria.

\section{MODELOS BASADOS EN AGENTES}

Los modelos basados en agentes son un formalismo cuyo origen puede remontarse a los autómatas celulares; por tanto, para comprender a los primeros, necesitamos saber, aunque sea mínimamente, qué son estos últimos.

Un autómata celular es un modelo matemático de un sistema dinámico ${ }^{(14)}$. Puede ser definido como una colección de celdas discretas y deterministas en hilera, en grilla o en $\mathrm{n}$ dimensiones, donde el valor de cada celda es un atributo binario (presencia o ausencia, prendido o apagado, vivo o muerto, verdadero o falso, etc.) y cuyo estado se actualiza a lo largo de un tiempo también discreto, en función de los estados de las celdas vecinas $^{(6)}$. Cuando hablamos de tiempo discreto nos referimos a que no es una magnitud continua para este tipo de modelos; es decir, es como los juegos por turnos, en los que cada jugador tiene un tiempo propio (como en el ajedrez). La colección, sea en hilera, en grilla o en más dimensiones puede tener un borde concreto o bien conectar los extremos entre sí. Esta segunda opción es la más utilizada y podemos vislumbrarla si pensamos en un tablero de pac man (donde los fantasmitas y el propio pac man, salían por un costado y volvían a entrar por el otro). La actualización se realiza en forma discreta y sincrónica; la vecindad suele ser ortogonal o puede incorporar también las diagonales (si estamos en presencia de una grilla, dos dimensiones); el radio de vecindad se define de antemano y puede ser de 1 (las celdas inmediatas) o n, dependiendo de los intereses del investigador.

En ciencias sociales, esta clase de modelos suelen ser llamados "de tablero". Existen algunos célebres, como el que desarrolló Thomas Schelling en $1969^{(6,15)}$, para modelizar problemas de segregación racial, en el que muestra que no es necesario ser un racista militante para lograr espacios geográficos diferenciados por grupo étnico (ghettos). Un modelo similar, pero desarrollado en forma independiente, fue el modelo de Sakoda, en el que intentaba observar el comportamiento de los campos de relocalización de japoneses en los EE.UU. durante la Segunda Guerra Mundial ${ }^{(6,16,17)}$.

Los modelos basados en agentes son una extensión de los autómatas celulares. En primer lugar, aparecen agentes que habitan sobre el tablero. Estos agentes pueden tener atributos diferentes y sus valores no están restringidos a valores binarios: pueden ser de la naturaleza que el investigador crea conveniente. En algún sentido virtual, entonces, los agentes habitan el tablero. En segundo lugar, el tablero tampoco está restringido en cada celda a dos valores binarios, sino que pueden predicarse, de cada una, la cantidad de variables que se crean convenientes y asignarles una naturaleza deseada (es decir, las variables pueden tener cualquier tipo de dato); esto hace que el tablero sea computacionalmente activo y pueda simularse prácticamente cualquier cosa en él.

Un ejemplo puede ilustrar mejor la potencia de los modelos basados en agentes. Tal vez, la implementación más famosa sea 
la del "Sugarscape", el modelo desarrollado por Joshua Epstein y Robert Axtell(18). Este sistema consta de un tablero en el que se distribuye en forma no aleatoria un recurso que se denomina "azúcar". La distribución sigue un patrón de círculos, donde lo más naranja, coincide con la mayor concentración del recurso, como se muestra en la Figura 3.

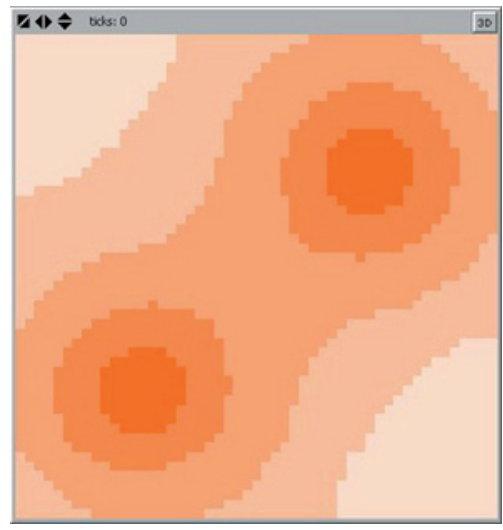

Figura 3. Visualización de la distribución del recurso (azúcar) en el tablero del modelo Sugarscape, según el entorno de modelado Netlogo.

Fuente: Centre for Interdisciplinary Science ${ }^{(19)}$.

En la Figura 3, la zona de color naranja oscuro indica que las celdas de esa región poseen más unidades de "azúcar" que las zonas con un color naranja más claro. Una vez consumido el recurso, dependiendo del interés del usuario, puede volver a crecer en forma inmediata, en forma gradual o no crecer en lo absoluto.

Sobre este tablero "viven" los agentes, quienes poseen una serie de propiedades: una capacidad de visión (medida en cantidad de celdas que pueden ver), un metabolismo (que indica la cantidad de "azúcar" que necesita cada agente para no morir de inanición) y una edad (que se actualiza en cada "turno" del sistema y que, llegada a un límite sorteado entre los agentes al azar, determina su muerte). En función de las propiedades del tablero o mundo y de los agentes, se montan un conjunto de reglas que indican tanto la conducta de los agentes como la conducta del mundo. Estas reglas indican, por ejemplo, cuánto debe crecer el azúcar, una vez que fue consumida, e indican también cómo los agentes buscarán, en función de su capacidad de visión, en cuál celda existe la mayor concentración de azúcar, irán hacia allí y consumirán todo el recurso que encuentren. Lo que no es consumido por su metabolismo, se almacenará. En la Figura 4, puede observarse un ejemplo del tablero con los agentes.

Este modelo, con su simplicidad manifiesta, permite simular fenómenos complejos. Por ejemplo, los autores muestran cómo se produce una distribución desigual de la riqueza, o cómo se puede refutar modélicamente el darwinismo social, o bien cómo puede propagarse una enfermedad o generarse una identidad cultural ${ }^{(18)}$. Una de las claves de este tipo de modelos es que permite jugar con la heterogeneidad de los agentes y del entorno. A diferencia de los modelos estadísticos que exigen cierta homogeneidad para que las medidas tengan significado, o los modelos cualitativos que enfatizan en la diversidad, los modelos basados en agentes permiten aprovechar lo mejor de ambos mundos, presentándose como un espacio excelente para someter a prueba las hipótesis

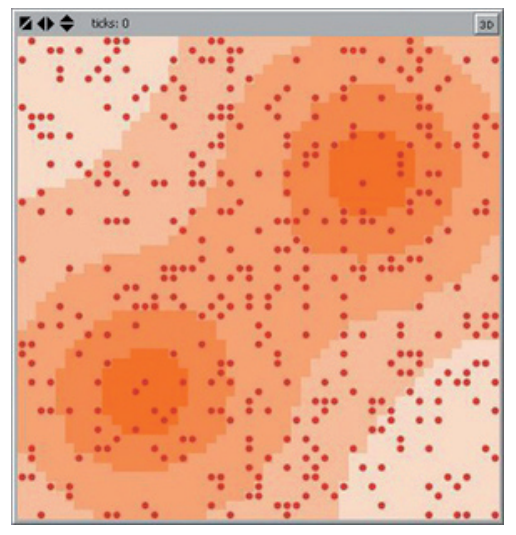

Figura 4. Visualización de la distribución de los agentes y del recurso (azúcar) en el tablero del modelo Sugarscape, según el entorno de modelado Netlogo.

Fuente: Centre for Interdisciplinary Science ${ }^{(19)}$. 
cualitativas y observar cómo, a partir de las interacciones locales, se generan los grandes resúmenes estadísticos. En definitiva, este tipo de modelos no son ni cuantitativos ni cualitativos, se ubican en una transición de fase, en ese espacio metodológico intermedio. Tal como menciona Reynoso:

Los MBA [modelos basados en agentes], con sus hormiguitas y sus premios en azúcar, tienen sin duda cierto toque de estupidez, como si la vida real les quedara grande; pero, en el proceso de su tratamiento, uno se ve forzado a reflexionar sobre las implicancias desbordantes de cualquier enunciado sobre un mundo cultural regido por principios de no linealidad. ${ }^{(6)}$

\section{Un modelo basado en agente sobre intercambio alimentario en la Quebrada de Humahuaca}

El modelo que desarrollamos y presentamos aquí fue el resultado del trabajo de campo realizado en la Quebrada de Humahuaca ${ }^{(8)}$, que sirvió como insumo para poder abstraer ciertas reglas del comportamiento alimentario, vinculadas específicamente con la reciprocidad y el intercambio de alimentos y aplicarlas al modelo basado en agente.

Cuando se trabaja con una sociedad artificial es prioritario tener en cuenta el recorte que se realiza de la realidad. La ficción holística de la escritura etnográfica se hace añicos frente a las restricciones que impone la computadora. El diseño de un modelo de esta clase exige que se tomen algunas decisiones metodológicas, ya que no todo puede ni debe simularse, sino que debe aplicarse un criterio de selección que se adecue al conjunto de hipótesis elegido.

La gran ventaja que presentan las sociedades artificiales - uno de los nombres de los modelos basados en agentes utilizados para trabajar con datos sociales- es que permiten que cada una de las unidades que se toman como protagonista de la simulación -en nuestro caso, las unidades domésticaspuedan ser diferentes entre sí. La variabilidad de cada uno de los agentes es una de las claves de la potencialidad y es lo que aleja a esta metodología de los presupuestos que rigen los modelos estadísticos ${ }^{(8)}$, en los que necesariamente se reduce la diversidad de los sujetos en beneficio de medidas que resumen todos los datos, como el promedio o el desvío estándar. Este atributo de la diversidad es, a su vez, el que acerca estos modelos a la mirada etnográfica. La ventaja que tienen las sociedades artificiales sobre la etnografía es la posibilidad de observar en tiempo real (en términos computacionales) la dinámica del fenómeno que se está investigando, así como reproducirla, confirmando las observaciones del mundo real o sugiriendo nuevos vínculos y conexiones.

Desde nuestra perspectiva, las sociedades artificiales son un complemento del equipaje metodológico que porta el antropólogo, que agrega dinamismo y síntesis sobre la variabilidad etnográfica (usada como insumo) y, a la vez, tiene la capacidad de expresar y describir los resultados de esa dinámica en términos estadísticos, si esto fuera necesario.

Nos atrevemos a señalar que las sociedades artificiales pueden ocupar el papel del complemento operativo y metodológico de los conceptos teóricos vinculados con el habitus $^{(20)}$ o bien con la agencia ${ }^{(21)}$, en tanto permiten ver (en un entorno controlado) cómo se desarrollan y cuánto alcance tienen las acciones de los sujetos (sean estos individuos, unidades domésticas o cualquier otro agregado de interés).

Aquí intentamos observar la dinámica del consumo de dos clases de comidas (industriales y locales) en relación con dos factores: 1) la provisión de alimentos mediante la compra o la producción y 2) la provisión de alimentos mediante la reciprocidad y el intercambio con vecinos y familiares.

Si tomamos el concepto de "estrategias domésticas de consumo"(22) podremos observar que entre sus dimensiones se encuentra la "diversificación de las fuentes de abastecimiento". Esto quiere decir que las fuentes en las que las unidades domésticas 
se proveen de comida son diversas y no están un solo lugar. En este sentido, una de las fuentes a considerar es la del intercambio de alimentos en el ámbito de la familia ampliada (entendiendo también dentro de esta a los amigos íntimos). Dicho de otro modo, el abasto de alimentos no pasa únicamente por los comercios de diferentes tipos en los que se compra la mercadería, sino que incluye tanto las prestaciones que brinda el Estado, como otras instituciones no públicas, así como también los intercambios alimentarios por fuera del sistema formal.

En esas relaciones informales entre familiares circulan alimentos provenientes de la producción rural de la Quebrada, y esto es lo que quisimos representar en este modelo basado en agentes.

Pero el origen de los alimentos, su pertenencia a la industria o a la producción local, recién se hizo evidente cuando analizamos los datos que obtuvimos durante el trabajo de campo, ya que no es un dato que se pueda encontrar o deducir de las estadísticas oficiales. En las redes sociales que mostramos en el apartado anterior, registradas en el ámbito urbano, vimos que hay una presencia importante, aunque no total, de los alimentos industriales. Aparecieron también, en un lugar destacado, algunos alimentos que consideramos locales, como los panes caseros o bien el queso de cabra. El ámbito urbano no se correspondió en un ciento por ciento con una alimentación industrial, sino que aparecieron también producciones alimentarias locales. En las entrevistas también quedó en claro la complejidad del sistema alimentario. Fue allí donde se revelaron, en toda su magnitud, la influencia de la producción local y de los circuitos alternativos al mercado capitalista, que forman parte de las estrategias de consumo de las unidades domésticas.

Esta dinámica es la que intentamos replicar en nuestra sociedad artificial: la alimentación industrial y la local distribuidas de manera heterogénea dentro de la ubicación espacial, donde se observan alimentos industriales en el área rural y aparecen producciones locales en el área urbana.
Para su reproducción in silico (simulada) nos basamos en las reglas de comportamiento alimentario que observamos en el trabajo de campo, en el contacto cotidiano con la población.

Asumimos, para nuestro modelo basado en agentes, que las unidades domésticas no se diferenciaban por sus ingresos sino por sus posibilidades: por consumir alimentos industriales o bien alimentos de producción local. En esta sociedad artificial recortamos a todas aquellas unidades domésticas que poseían ingresos elevados y que, por lo tanto, tenían un margen de maniobra mayor en cuanto a la satisfacción de sus necesidades alimentarias. Poseer ingresos altos permite que, si bien la demanda alimentaria es siempre inelástica (tiende a un límite) (22), las posibilidades de elección (y de previsión) se multiplican en función de ese mismo ingreso. En el mundo que creamos, la desigualdad no estaba dada por los ingresos o por la propiedad, sino por la ubicación geográfica. Sabemos que creamos una ficción, pero en todo caso, una ficción interesada y acorde a los objetivos de un aspecto de la investigación. Esta flexibilidad de las sociedades artificiales es uno de sus más valiosos aportes.

Pasemos pues a describir nuestro sistema. En la Figura 5 mostramos una pantalla en la que se observa un tablero dividido en dos áreas, una verde y otra gris. Dentro del área verde hay unos cuadrados grises que se distribuyen en la parte inferior del área verde. Distribuidos por todo el tablero en forma aleatoria hay unas casitas, que representan nuestras unidades domésticas. El área verde representa la zona rural, mientras que el área gris representa el área urbana o, mejor dicho, el área donde es posible conseguir alimentación industrial. Cada casita es una unidad doméstica que posee unas aristas que salen de ella y se conectan con otras casitas distribuidas por el tablero. Estos lazos representan los vínculos sociales de las unidades domésticas, las cuales, en algunos casos, poseen un lazo; en otros, dos; en unos pocos, tres vínculos; y hay otros poquitos con más de tres vínculos. La regla que asigna esos lazos es la del azar. Se recorren todas las unidades 
domésticas y a cada una se le asigna un lazo con otra unidad doméstica al azar. La restricción es que una unidad doméstica no puede tener un lazo consigo misma. No olvidemos que el pseudo random que utiliza la computadora suele tener una distribución gaussiana. Decidimos que la mejor manera de establecer los vínculos era el azar, en función de que consideramos que esa distribución se ajusta a lo observado en el campo; es decir, si bien en la realidad la gente no establece sus lazos al azar, hay toda una historia que determina los tipos de vínculos, lo cierto es que la configuración final (para nuestros objetivos) es muy similar a la que plantea la aleatoriedad, sobre todo porque aquí tomamos como fundamento de los vínculos la posibilidad de intercambiar alimentos en una dirección prefijada, que va del campo a la ciudad y no necesariamente a las relaciones sociales en su totalidad. Hay ciertos estudios que plantean, por ejemplo, que los vínculos sexuales o los lazos de amistad siguen una ley de potencia en una sociedad ${ }^{(7,12)}$. En nuestras observaciones, no hemos encontrado que la gente del ámbito urbano envíe alimentos industriales a sus contactos en el campo, pero sí observamos lo opuesto: mucha gente con vínculos en el área rural se trae de allí algunos alimentos que siempre son producidos localmente.

La alimentación industrial en el campo está dada por la cercanía con el ámbito urbano (el área gris de nuestro modelo) y por la cercanía con los negocios que se encuentran distribuidos por la zona rural (los cuadrados grises) (ver Figura 5). A partir de esta idea es que tomamos la distancia a esos lugares y en función de ellos aplicamos otra vez un criterio azaroso: a medida que una

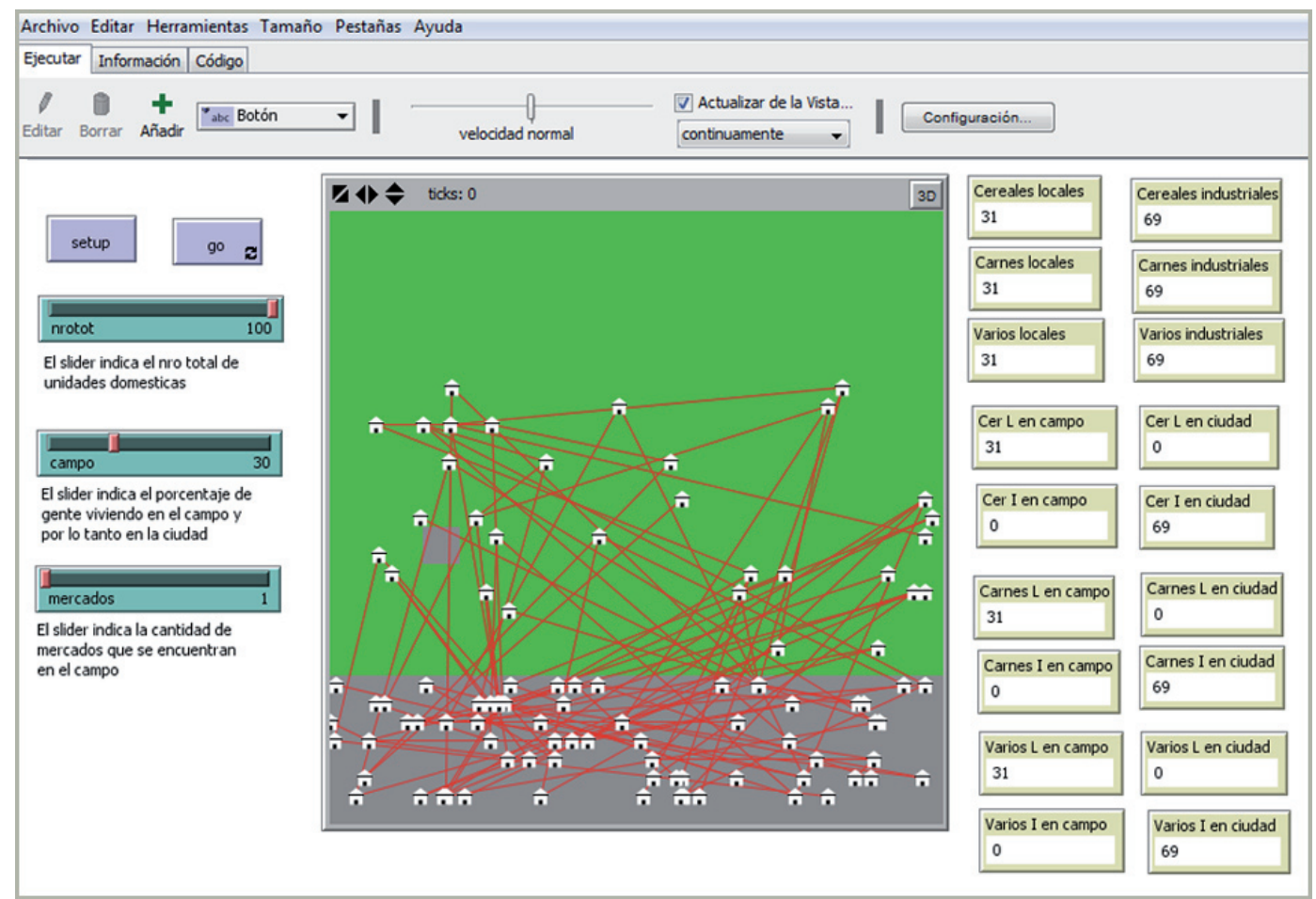

Figura 5. Visualización de la distribución de las unidades domésticas en el área rural y el área urbana, en la interfaz de NetLogo. Quebrada de Humahuaca, Jujuy, Argentina. 
unidad doméstica se acerca a alguna de las áreas grises, la probabilidad de obtener alimentos industriales es mayor.

Entre los controles que se observan en la interfaz (Figura 5) está el botón "setup", que es el que configura el escenario, ejecuta las rutinas que crean el área urbana, la rural y los negocios, crea y distribuye las unidades domésticas, así como sus lazos. También en la función "setup" se asignan los alimentos que poseen por defecto las unidades domésticas. Siempre se comienza con una distribución en la que las unidades domésticas urbanas cuentan con alimentos exclusivamente industriales, mientras que las rurales arrancan con alimentos producidos solo localmente. El botón denominado "go" pone en marcha el sistema en forma permanente, es decir, no ejecuta una vuelta y frena, sino que continúa hasta que el usuario decida terminar la ejecución. En la rutina "go" las unidades domésticas intercambian alimentos con sus vínculos en el campo y luego se proveen de alimentos: si las unidades domésticas están ubicadas en el área verde, entonces, como ya fue dicho, la distancia al área gris es la que determina la probabilidad de obtener alimentos industriales; si no se cumple, entonces se proveen de alimentos producidos localmente.

Los alimentos fueron aquí definidos como de tres tipos para dos variantes. Dividimos el patrón alimentario en cereales (que incluye todo tipo de vegetales); carnes (todos los tipos de carnes) y varios (que incluye todo lo demás). Esta clasificación está a su vez dividida en locales o industriales. Toda esta simplificación con respecto a los datos de la realidad tiene su justificación en el objetivo del modelo planteado, que es observar en forma dinámica cómo estos se van distribuyendo de acuerdo a las estrategias de provisión de las propias unidades domésticas: por un lado, los intercambios, por el otro, el autoconsumo y, por último, la posibilidad de obtener los alimentos industriales en el mercado formal.

La interfaz cuenta con un control denominado "nrotot" que define la cantidad de unidades domésticas que se dibujarán en el mundo. Tenemos también otro control que se denomina "campo" que define los porcentajes de gente que vive tanto en el área rural como en el área urbana. En la Figura 5 vemos que ese valor está en 30, lo que significa que un $70 \%$ de toda la población vive en el área urbana, mientras que el $30 \%$ restante vive en el área rural. Por último tenemos un control que se llama "mercados" que indica la cantidad de negocios que puede haber en el campo, en esta versión las posibilidades van de 1 a 6 .

En la Figura 5, a la derecha del escenario, vemos que la interfaz cuenta con una serie de controles que nos permiten monitorear qué es lo que está sucediendo con los alimentos en cada vuelta (cada tick). Los seis primeros muestran la distribución general de alimentos, divididos en locales e industriales y por categoría de alimentos. Los 12 siguientes nos muestran esos mismos alimentos pero en su ocurrencia de acuerdo al ámbito, así tenemos diferenciados los distintos alimentos locales e industriales, tanto en el campo como en la ciudad.

Como ya fue señalado, comenzamos las corridas (es decir la ejecución del programa) con una distribución que se corresponde con la hipótesis que planteamos en un comienzo, esto es, que todos los que viven en el ámbito urbano consumen alimentos industriales y que todos los que viven en el ámbito rural consumen alimentos locales. En la Figura 6 se visualiza lo que ocurre al cabo de unas cuantas vueltas (ticks), en este caso 10.192. La distribución se estabiliza y aparecen, tanto en la ciudad como en el campo, alimentos de una y otra categoría. Claro, siempre con un sesgo: en la ciudad hay más alimentos industriales, mientras que en el área rural hay más alimentos de producción local. ¿Qué nos muestra esto? Por un lado, que el patrón alimentario, al igual que ocurre en la realidad, se encuentra diferenciado y no es homogéneo, como habíamos supuesto en un primer momento $y$, por otro, que una de las claves se encuentra en los procesos de intercambio motorizados por la reciprocidad, que permite que haya una cierta afluencia de alimentos producidos localmente hacia la urbe. Es interesante hacer notar que, en nuestro modelo, la única manera de obtener 


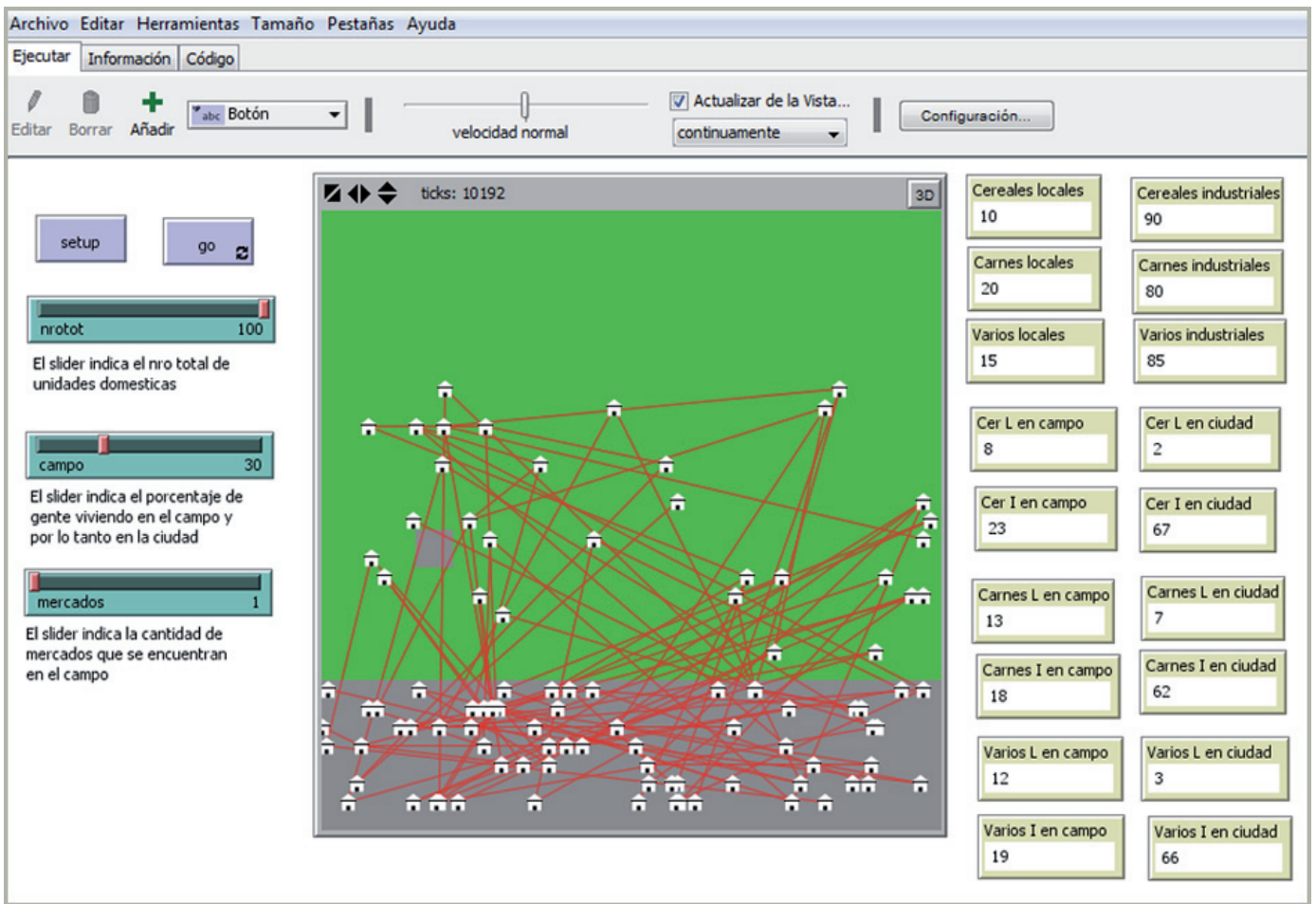

Figura 6. Visualización de la distribución de las unidades domésticas en el área rural y el área urbana, luego de 10.192 corridas (ticks) en la interfaz de NetLogo. Quebrada de Humahuaca, Jujuy, Argentina

Fuente: Díaz Córdova ${ }^{(8)}$.

alimentos producidos localmente es a partir de los lazos que las unidades domésticas mantienen entre sí y no comprándolos en algún mercado de la ciudad, como observamos que ocurre muchas veces en la Quebrada (una clara diferencia del modelo con la realidad).

Lo que quisimos poner a prueba con este modelo era la posibilidad de reproducir, con todas las cautelas del caso, algunas actitudes y comportamientos alimentarios de los pobladores quebradeños, y reflexionar sobre la información que devuelve el campo, pero desde una perspectiva que no admite juegos retóricos, sino que impone una definición concreta, aunque sea operativa y coyuntural. Los modelos basados en actores no son una panacea, no sustituyen otras metodologías; nos permiten verificar si nuestras hipótesis tienen algún rastro de realidad o al menos de plausibilidad. Nos permiten conocer, desde otro punto de vista, los fenómenos bajo estudio. La clave, como fue dicho, no está en el dominio técnico, sino en la mirada epistemológica; en la posibilidad de materializar ese modelo mental que siempre ocurre y que, por su carácter inevitable, siempre está presente. Lo mejor es tratar a los modelos, simplemente, como forma de pensar.

\section{CONCLUSIONES}

Hemos intentado, hasta aquí, presentar dos metodologías no muy utilizadas en el ámbito de la antropología alimentaria. Por un lado, el análisis de redes sociales, por el otro, los modelos basados en agentes. Estas dos formas de mirar al mundo se enrolan dentro de las corrientes teóricas de la complejidad y 
el caos. Dados los indicios que muestran que el universo y todo lo que está dentro es complejo, entonces qué mejor que utilizar herramientas que, con las limitaciones del caso, den cuenta de esa complejidad manifiesta. La antropología alimentaria es un campo fértil para la experimentación metodológica; como la alimentación es un "hecho social total"(1) y su propio estudio es multidisciplinar por naturaleza, la experimentación metodológica se torna necesaria. No podemos resignar métodos por supuestos y prejuicios. Hay que someterlos a prueba y verificar sus alcances y limitaciones. Estar dispuestos a modificar el punto de vista, adaptarlo a las exigencias del método, sin forzar tampoco su propia arquitectura. Un justo medio aristotélico que solo puede conseguirse en la praxis.

El análisis de redes sociales, en nuestra experiencia, tiene la ventaja de clarificar ciertas estructuras subyacentes, de las que se sospecha su existencia o no. Permite, por un lado, corroborar o refutar las hipótesis pero, por el otro, es también una herramienta de descubrimiento. En nuestro caso, a partir del trabajo de campo, empezó a hacerse evidente que las modalidades alimentarias (industrial y local) no se encontraban separadas en el ámbito urbano, no al menos de la forma en que habíamos sospechado en un comienzo. Fue por ello que decidimos aplicar el análisis de redes sociales a los datos que ya teníamos, lo cual también es interesante, dado que puede ser usado tanto con datos que no fueron pensados en términos de redes, como con datos recolectados ex profeso. Así transformamos los recordatorios alimentarios de 24 hs en datos reticulares, susceptibles de ser analizados con las herramientas clásicas del análisis de redes sociales, para ver cuál alimento tenía más conexiones, a qué distancia estaba cada alimento respecto del resto, qué grado de cohesión tenía la red en su conjunto, etc.

Una de las desventajas más claras que posee el análisis de redes sociales es que los análisis son sincrónicos, es decir, toman una foto de un momento puntual del tiempo. $\mathrm{Si}$ bien muchas corrientes teóricas como el estructuralismo o el funcionalismo utilizan un enfoque sincrónico, eso no quita que sus posibilidades se vean, en un sentido, limitadas por esa característica. En el caso del análisis de redes sociales, existe una posibilidad metodológica de observar la trayectoria de una red a lo largo del tiempo aunque, hay que decirlo, posee cierta complicación algorítmica. En cualquier caso, esta limitación muestra cuál es el alcance de la metodología, impidiendo confusiones teóricas al respecto ${ }^{(11)}$.

Con respecto a los modelos basados en agentes, creemos que la principal ventaja radica en la forma de pensar los problemas, que es una exigencia de este tipo de metodologías. Aquí lo central es escoger qué actores (o agentes) serán los importantes y qué características tendrán. Lo mismo para el escenario construido, así como para las relaciones entre actores y entre actores y medioambientes. En nuestro caso, nos interesó simular las relaciones reciprocitarias vinculadas con los alimentos, en un contexto como el quebradeño, en el que conviven mercado y producción local. Es decir, nos interesó implementar computacionalmente una de las prácticas que conforman las "estrategias domésticas de consumo"(22); en este caso, la diversificación de las fuentes de abasto en su aspecto particular del intercambio reciprocitario de comida. Quisimos reproducir esa dinámica del intercambio, tal como la vimos en el campo: esa síntesis entre lo que observamos, participamos y registramos de lo que dicen y hacen, y dicen que hacen los actores. Quedó bastante claro que el patrón reciprocitario convive con el mercado y que los alimentos locales tienen cierto poder de resiliencia.

Los modelos basados en actores ayudan a conocer la dinámica de lo que acontece en el entorno social estudiado. Una de sus virtudes es que nos permiten jugar con diferentes características y distintas configuraciones, son una herramienta para conocer el espacio etnográfico desde una perspectiva diferente, en la cual el control está claramente del lado de quien construye el modelo (actores sociales e investigador). En ese sentido, hay ya autores que hablan de "simulación participante"(23), entendiendo algo similar a la observación 
participante, pero aplicado a los modelos basados en agentes ${ }^{(11)}$.

La gran desventaja de esta clase de modelos es que exigen cierta pericia algorítmica. En algún sentido, conocer las posibilidades del lenguaje de programación en el que se desarrollará permite apreciar mejor las posibilidades que brinda. Otro de los peligros que se detectan es el de la "falacia de la realidad", que consiste en intentar simular todo lo que ocurre en el campo, generando un modelo tan complicado que puede atentar contra la ventaja de la simplicidad. La clave para poder usar correctamente los modelos basados en actores es escoger y abstraer aquellos elementos de la realidad que, por algún motivo, se consideran relevantes, sea porque los actores hacen referencia, sea porque el marco teórico del investigador así lo indica.

\section{REFERENCIAS BIBLIOGRÁFICAS}

1. Mauss M. Ensayo sobre el don: Forma y función del intercambio en las sociedades arcaicas. Buenos Aires: Katz Editores; 2009.

2. Hanneman R, Riddle M. Introduction to social networks methods [Internet]. Riverside: University of California; 2005 [citado 10 jul 2016]. Disponible en: http://tinyurl.com/ju54p9b.

3. Wilensky $U$, Rand B. An introduction to agentbased modeling: modeling natural, social, and engineered complex systems with NetLogo. Cambridge: MIT Press; 2015.

4. Merton R. Teoría y estructuras sociales. México: Fondo de Cultura Económica; 1980.

5. Agar M. Agents in living color: Toward emic agent-based models. Journal of Artificial Societies and Social Simulation [Internet]. 2005;8(1) [citado 10 jul 2016]. Disponible en: http://tinyurl.com/ hjm5rwd.

6. Reynoso C. Complejidad y caos: Una exploración antropológica. Buenos Aires: SB; 2006.
7. Watts D. Seis grados de separación: La ciencia de las redes en la era del acceso. Barcelona: Paidós; 2006.

8. Díaz Córdova D. Antropología alimentaria de la Quebrada de Humahuaca: Modos de producción, patrón alimentario y sus efectos sobre el cuerpo y la salud de la población quebradeña. [Tesis de Doctorado]. Buenos Aires: Universidad de Buenos Aires; 2015.

9. Muscio H. Colonización humana del NOA y variación en el consumo de los recursos: La ecología de los cazadores recolectores de la Puna durante la transición Pleistoceno-Holoceno. Naya [Internet]. s/f [citado 10 jul 2016]. Disponible en: http://tinyurl.com/hm5kngw.

10. Reboratti C. La Quebrada: Un estudio geográfico, histórico y social de este Patrimonio de la Humanidad. Buenos Aires: La Colmena; 2003.

11. Antropocaos. Exploraciones en antropología y complejidad. Buenos Aires: SB; 2009.

12. Reynoso C. Redes sociales y complejidad: Modelos interdisciplinarios en la gestión sostenible de la sociedad y la cultura. Buenos Aires: SB; 2011. 
13. Wasserman S, Faust K. Social networks analysis: methods and applications. New York: Cambridge University Press; 1994.

14. Wolfram S. Cellular automata. Los Alamos Science. 1983;9:2-21.

15. Schelling T. Models of Segregation. The American Economic Review. 1969;59(2):488-493.

16. Schelling T. Dynamic models of segregation. Journal of Mathematical Sociology. 1971;1:143-186.

17. Sakoda J. The checkerboard model of social interaction. Journal of Mathematical Sociology. 1971;1:119-132.

18. Epstein J, Axtell R. Growing artificial societies: Social Science from the Bottom Up. Washington DC: Brookings Institution Press; 1996.
19. Centre for Interdisciplinary Science. Complex Systems-Sugarscape [Internet]. University of Leicester [citado 10 jul 2016]. Disponible en: http:// tinyurl.com/z6k34os.

20. Bourdieu P. Razones prácticas sobre la teoría de la acción. Barcelona: Anagrama; 1997.

21. Giddens A. La constitución de la sociedad: Bases para la teoría de la estructuración. Buenos Aires: Amorrortu; 2006.

22. Aguirre P. Estrategias de consumo: qué comen los argentinos que comen. Buenos Aires: Miño y Dávila; 2015.

23. Eglash R. African fractals: Modern computing and indigenous design. New Brunswick: Rutgers University Press; 1999.

\section{FORMA DE CITAR}

Díaz Córdova D. Novedades metodológicas aplicadas a la antropología alimentaria: modelos basados en agentes y redes sociales. Salud Colectiva. 2016;12(4):635-650. doi: 10.18294/sc.2016.1008.

Recibido: 13 de junio de 2016 | Versión final: 22 de septiembre de 2016 | Aprobado: 16 de noviembre de 2016

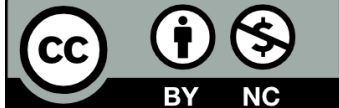

Este obra está bajo una licencia de Creative Commons Reconocimiento-NoComercial 4.0 Internacional. Reconocimiento - Permite copiar, distribuir y comunicar públicamente la obra. A cambio, se debe reconocer y citar al autor original. No Comercial - Esta obra no puede ser utilizada con finalidades comerciales, a menos que se obtenga el permiso.

http://dx.doi.org/10.18294/sc.2016.1008 